\title{
DENSITY TOPOLOGIES ON THE PLANE BETWEEN ORDINARY AND STRONG. II
}

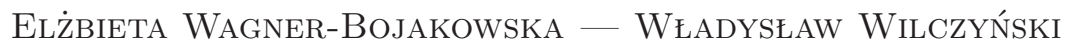

\begin{abstract}
Let $C_{0}$ denote a set of all non-decreasing continuous functions $f:(0,1] \rightarrow(0,1]$ such that $\lim _{x \rightarrow 0^{+}} f(x)=0$ and $f(x) \leq x$ for every $x \in(0,1]$, and let $A$ be a measurable subset of the plane. The notions of a density point of $A$ with respect to $f$ and the mapping $D_{f}$ defined on the family of all measurable subsets of the plane were introduced in Wagner-Bojakowska, E.-Wilczyński, W.: Density topologies on the plane between ordinary and strong, Tatra Mt. Math. Publ. 44 (2009), 139-151. This mapping is a lower density, so it allowed us to introduce the topology $\mathcal{T}_{f}$, analogously to the density topology. In this note, properties of the topology $\mathcal{T}_{f}$ and functions approximately continuous with respect to $f$ are considered. We prove that $\left(\mathbb{R}^{2}, \mathcal{T}_{f}\right)$ is a completely regular topological space and we study conditions under which topologies generated by two functions $f$ and $g$ are equal.
\end{abstract}

Let $\mathcal{S}$ denote a family of all Lebesgue measurable subsets of the plane and $m_{2}$ the Lebesgue plane measure. For $A \in \mathcal{S}$, let $\Phi_{0}(A)$ and $\Phi_{s}(A)$ denote a set of all ordinary density points of $A$ and a set of all strong density points of $A$, respectively (see [2]).

If $A, B \in \mathcal{S}$, then we shall write $A \sim B$ if and only if $m_{2}(A \triangle B)=0$ (where $A \triangle B=(A \backslash B) \cup(B \backslash A)$ ).

Let $C_{0}$ denote a set of all non-decreasing continuous functions $f:(0,1] \rightarrow(0,1]$ such that $\lim _{x \rightarrow 0^{+}} f(x)=0$ and $f(x) \leq x$ for every $x \in(0,1]$.

Let $f \in C_{0}$ and $A \in \mathcal{S}$.

Definition 1 ([3]). We shall say that $(0,0)$ is a density point of $A$ with respect to a function $f$ if for each $\epsilon>0$ there exists $\delta>0$ such that for each $h, k \in(0, \delta)$ if $f(h) \leq k \leq h$ or $f(k) \leq h \leq k$, then

$$
\frac{m_{2}(A \cap\{[-h, h] \times[-k, k]\})}{4 h k}>1-\epsilon,
$$

(C) 2015 Mathematical Institute, Slovak Academy of Sciences.

2010 Mathematics Subject Classification: Primary 28A05; Secondary 54A05, 54A10, $54 \mathrm{D} 15$.

Keywords: density point, density topology, density point with respect to $f$.

The research was supported by the grant N N201 547238 . 


\section{ELŻBIETA WAGNER-BOJAKOWSKA — WŁADYSŁAW WILCZYŃSKI}

or, equivalently,

$$
\frac{m_{2}\left(A^{\prime} \cap\{[-h, h] \times[-k, k]\}\right)}{4 h k}<\epsilon
$$

where $A^{\prime}=\mathbb{R}^{2} \backslash A$.

We say that $\left(x_{0}, y_{0}\right)$ is a density point of $A$ with respect to $f$ if $(0,0)$ is a density point of $A-\left(x_{0}, y_{0}\right)=\left\{\left(x-x_{0}, y-y_{0}\right):(x, y) \in A\right\}$ with respect to $f$.

For $A \in \mathcal{S}$ and $f \in C_{0}$, by $D_{f}(A)$ we shall denote a set of all points $(x, y) \in \mathbb{R}^{2}$ which are density points of $A$ with respect to $f$. Clearly, $\Phi_{s}(A) \subset D_{f}(A) \subset \Phi_{0}(A)$ for every $A \subset \mathcal{S}$. The mapping $D_{f}: \mathcal{S} \rightarrow 2^{\mathbb{R}}$ is a lower density (see [3], Theorem 5]), so the family

$$
\mathcal{T}_{f}=\left\{A \in \mathcal{S}: A \subset D_{f}(A)\right\}
$$

is a topology on the plane, essentially stronger than the strong density topology $\mathcal{T}_{s}$ on the plane and weaker than the ordinary density topology $\mathcal{T}_{0}$ on the plane. The properties of the topology $\mathcal{T}_{f}$ were studied in [3], (see Theorems $7-15$ ). Among others, there was proved that $\left(\mathbb{R}^{2}, \mathcal{T}_{f}\right)$ is a regular $\left(T_{3}\right)$ Baire space. In this note we shall show that $\left(\mathbb{R}^{2}, \mathcal{T}_{f}\right)$ is a completely regular $\left(T_{3 \frac{1}{2}}\right)$ topological space. For this purpose, observe that the theorem analogous to Lusin-Menchoff theorem holds. The proof of the next theorem is analogous to the proof of Lusin-Menchoff theorem in [2] with necessary changes.

Theorem 1. Let $E \in \mathcal{S}$ and let $X$ be a closed subset of the plane such that $X \subset E \cap D_{f}(E)$. Then, there exists a perfect set $P \subset E$ such that

$$
X \subset P \cap D_{f}(P) \text {. }
$$

Proof. As $D_{f}(E) \subset \Phi_{0}(E)$, using in [1, Lemma 6.3, Chapter II], we obtain that there exists a perfect set $P_{0}$ such that $X \subset P_{0} \subset E$. Additionally, we may suppose that $E \subset\left[-\frac{1}{2}, \frac{1}{2}\right] \times\left[-\frac{1}{2}, \frac{1}{2}\right]$. Put

and

$$
Q_{n}=\left\{(x, y) \in \mathbb{R}^{2}: \frac{1}{n+1}<\operatorname{dist}\left((x, y), P_{0}\right) \leq \frac{1}{n}\right\}
$$

$$
S_{n}=Q_{n} \cap E
$$

for every $n \in \mathbb{N}$, where the distance $\operatorname{dist}\left((x, y), P_{0}\right)$ of a point $(x, y)$ from a set $P_{0}$ is considered in the metric $\rho$ defined as follows

$$
\rho\left(\left(x_{1}, y_{1}\right),\left(x_{2}, y_{2}\right)\right)=\max \left(\left|x_{1}-x_{2}\right|,\left|y_{1}-y_{2}\right|\right)
$$

for all $\left(x_{1}, y_{1}\right),\left(x_{2}, y_{2}\right) \in \mathbb{R}^{2}$. Obviously, $E=P_{0} \cup \bigcup_{n=1}^{\infty} S_{n}$. For each $n \in \mathbb{N}$, let $P_{n}$ be a perfect subset of $S_{n}$ such that

$$
m_{2}\left(S_{n} \backslash P_{n}\right)<\frac{1}{2^{n}} \cdot f\left(\frac{1}{n+1}\right) .
$$


Put

$$
P=P_{0} \cup \bigcup_{n=1}^{\infty} P_{n}
$$

It is easy to see that $P$ is perfect and $X \subset P \subset E$. Now, we shall prove that each point of $X$ is a density point of $P$ with respect to $f$. Let $(x, y) \in X$ and let $\left\{I_{j}\right\}_{j \in \mathbb{N}}$ be a sequence of intervals centered at $(x, y)$ such that

and if

$$
\operatorname{diam}\left(I_{j}\right) \underset{j \rightarrow \infty}{\longrightarrow} 0, \quad \operatorname{diam}\left(I_{j}\right) \leq 1 \quad \text { for } j \in \mathbb{N},
$$

$$
I_{j}=\left(x-h_{j}, x+h_{j}\right) \times\left(y-k_{j}, y+k_{j}\right), \quad \text { then } \quad f\left(h_{j}\right) \leq k_{j} \leq h_{j}
$$

or

$$
f\left(k_{j}\right) \leq h_{j} \leq k_{j} \quad \text { for } j \in \mathbb{N} .
$$

Let $j$ be a fixed positive integer. There are two cases:

$1^{o} I_{j} \cap S_{n}=\emptyset$ for each $n \in \mathbb{N}$. Then, $I_{j} \cap E=I_{j} \cap P_{0}$, and consequently,

$$
m_{2}\left(E \cap I_{j}\right)=m_{2}\left(P_{0} \cap I_{j}\right) \leq m_{2}\left(P \cap I_{j}\right) .
$$

$\mathbf{2}^{o} I_{j} \cap S_{n} \neq \emptyset$ for some $n \in \mathbb{N}$. Put $n_{j}=\min \left\{n \in \mathbb{N}: I_{j} \cap S_{n} \neq \emptyset\right\}$. Then, $I_{j} \cap S_{n}=\emptyset$ for $n<n_{j}$ and $I_{j} \cap S_{n_{j}} \neq 0$, so

$$
(E \backslash P) \cap I_{j} \subset \bigcup_{n=n_{j}}^{\infty}\left(S_{n} \backslash P_{n}\right)
$$

and, consequently, using (1), we obtain

$$
\begin{aligned}
m_{2}\left(E \cap I_{j}\right) & =m_{2}\left(P \cap I_{j}\right)+m_{2}\left((E \backslash P) \cap I_{j}\right) \\
& \leq m_{2}\left(P \cap I_{j}\right)+\sum_{n=n_{j}}^{\infty} m_{2}\left(S_{n} \backslash P_{n}\right) \\
& <m_{2}\left(P \cap I_{j}\right)+f\left(\frac{1}{n_{j}+1}\right) \cdot \frac{1}{2^{n_{j}-1}} .
\end{aligned}
$$

So,

$$
m_{2}\left(P \cap I_{j}\right)>m_{2}\left(E \cap I_{j}\right)-f\left(\frac{1}{n_{j}+1}\right) \cdot \frac{1}{2^{n_{j}-1}} .
$$

If $f\left(h_{n_{j}}\right) \leq k_{n_{j}} \leq h_{n_{j}}$, then $\frac{1}{n_{j}+1} \leq h_{n_{j}}$, so $f\left(\frac{1}{n_{j}+1}\right) \leq f\left(h_{n_{j}}\right) \leq k_{n_{j}}$, and consequently,

$$
m_{2}\left(I_{j}\right)=4 h_{j} k_{j} \geq 4 f\left(\frac{1}{n_{j}+1}\right) \cdot \frac{1}{n_{j}+1} .
$$

Analogously, if $f\left(k_{n_{j}}\right) \leq h_{n_{j}} \leq k_{n_{j}}$. Hence,

$$
\frac{m_{2}\left(P \cap I_{j}\right)}{m_{2}\left(I_{j}\right)}>\frac{m_{2}\left(E \cap I_{j}\right)}{m_{2}\left(I_{j}\right)}-\frac{f\left(\frac{1}{n_{j}+1}\right) \cdot \frac{1}{2^{n_{j}-1}}}{4 f\left(\frac{1}{n_{j}+1}\right) \cdot \frac{1}{n_{j}+1}}=\frac{m_{2}\left(E \cap I_{j}\right)}{m_{2}\left(I_{j}\right)}-\frac{n_{j}+1}{2^{n_{j}+1}} .
$$


Clearly, $n_{j} \underset{j \rightarrow \infty}{\longrightarrow} \infty$, so

$$
\liminf _{j \rightarrow \infty} \frac{m_{2}\left(P \cap I_{j}\right)}{m_{2}\left(I_{j}\right)} \geq \liminf _{j \rightarrow \infty} \frac{m_{2}\left(E \cap I_{j}\right)}{m_{2}\left(I_{j}\right)}=1
$$

because $(x, y) \in X$ and $X \subset D_{f}(E)$. Consequently, $X \subset D_{f}(P)$.

For $X, E \in \mathcal{S}$ we shall write $X \subset \cdot E$ if $X \subset E \cap D_{f}(E)$, i.e., $X$ is contained in the interior of $E$ in the topology $\mathcal{T}_{f}$.

Corollary 1. Let $E \in \mathcal{T}_{f}$ and let $X$ be a closed subset of $E$. Then, there exists a perfect set $P$ such that

$$
X \subset \cdot P \subset \cdot E \text {. }
$$

Proof. Since $E \in \mathcal{T}_{f}$, so $E$ is measurable and $X$ is a closed subset such that $X \subset E \cap D_{f}(E)$. According to Theorem 1, there exists a perfect set $P$ such that $X \subset P \cap D_{f}(P)$ and $P \subset E$. Consequently, $X \subset P$ and $P \subset \cdot E$, because $E \subset \cdot E$.

Definition 2. We shall say that a function $g: \mathbb{R}^{2} \rightarrow \mathbb{R}$ is $f$-approximately continuous at $p_{0} \in \mathbb{R}^{2}$ if there exists a set $E \in \mathcal{S}$ such that $p_{0} \in E \cap D_{f}(E)$ and $g_{\mid E}$ is continuous at $p_{0}$. We shall say that a function $g: \mathbb{R}^{2} \rightarrow \mathbb{R}$ is $f$-approximately continuous if it is $f$-approximately continuous at each point $p \in \mathbb{R}^{2}$.

Obviously, $D_{f}(E) \subset \Phi_{0}(E)$ for each $E \in \mathcal{S}$, so each $f$-approximately continuous function is approximately continuous and, consequently, a Baire one function.

TheOREM 2. If $g_{1}$ and $g_{2}$ are $f$-approximately continuous at $p_{0}$, then $g_{1}+g_{2}$, $g_{1}-g_{2}, g_{1} \cdot g_{2}$ are also $f$-approximately continuous at $p_{0}$. If $g_{2}(p) \neq 0$ on some neighbourhood of $p_{0}$, then $g_{1} / g_{2}$ is $f$-approximately continuous at $p_{0}$.

Theorem 3. If $g_{1}: \mathbb{R}^{2} \rightarrow \mathbb{R}$ is $f$-approximately continuous at $p_{0}$ and $g_{2}: \mathbb{R} \rightarrow \mathbb{R}$ is continuous at $g_{1}\left(p_{0}\right)$, then $g_{2} \circ g_{1}$ is $f$-approximately continuous at $p_{0}$.

Observe that for our kind of continuity we obtain a result analogous to classical Denjoy theorem (see in [1, Theorem 5.2, Chapter II]).

Let $I=(a, b)$ be an arbitrary interval on the real line.

TheOREM 4. A function $g: I \times I \rightarrow \mathbb{R}$ is measurable if and only if it is $f$-approximately continuous a.e.

Proof. If $g$ is $f$-approximately continuous a.e., then it is approximately continuous a.e., so, by Denjoy theorem, it is measurable.

Suppose now that $g$ is measurable. Then, by Lusin theorem, for each $n \in \mathbb{N}$ there exists a closed set $F_{n} \subset(I \times I)$ such that $m_{2}\left(I \times I \backslash F_{n}\right)<\frac{1}{n}$ and $g_{\mid F_{n}}$ is continuous at each point $p \in F_{n}$. 
Put

$$
F=\bigcup_{n=1}^{\infty}\left(F_{n} \cap D_{f}\left(F_{n}\right)\right) .
$$

We shall prove that $m_{2}((I \times I) \backslash F)=0$ and $g$ is $f$-approximately continuous at each point $p \in F$. Obviously,

$$
\begin{aligned}
m_{2}((I \times I) \backslash F) & \leq m_{2}\left((I \times I) \backslash\left(F_{n} \cap D_{f}\left(F_{n}\right)\right)\right) \\
& \leq m_{2}\left((I \times I) \backslash F_{n}\right)+m_{2}\left(F_{n} \backslash D_{f}\left(F_{n}\right)\right)<\frac{1}{n}
\end{aligned}
$$

for each $n \in \mathbb{N}$, since $m_{2}\left(F_{n} \backslash D_{f}\left(F_{n}\right)\right)=0$. Consequently, $m_{2}((I \times I) \backslash F)=0$. Now, let $p \in F$. Then, there exists $n_{0} \in \mathbb{N}$ such that $p \in F_{n_{0}} \cap D_{f}\left(F_{n_{0}}\right)$ and $g_{\mid F_{n_{0}}}$ is continuous at $p$. Hence, $g$ is $f$-approximately continuous at $p$.

It is clear that the last theorem holds also in the case when the domain of $g$ is an open rectangle or the whole plane $\mathbb{R}^{2}$.

We can also characterize the $f$-approximately continuous functions in terms of associated sets (compare with [1, Theorem 5.6, Chapter II]). For this purpose, we need a following lemma.

LEMMA 1. If $\left\{E_{n}\right\}_{n \in \mathbb{N}}$ is a descending sequence of measurable sets such that $(0,0) \in D_{f}\left(E_{n}\right)$ for each $n \in \mathbb{N}$, then there exists a strictly decreasing sequence $\left\{l_{n}\right\}_{n \in \mathbb{N}}$ of positive numbers tending to zero such that

$$
(0,0) \in D_{f}\left(\bigcup_{n=1}^{\infty}\left(E_{n} \backslash\left\{\left[-l_{n}, l_{n}\right] \times\left[-l_{n}, l_{n}\right]\right\}\right)\right) .
$$

Proof. Let $\left\{\epsilon_{n}\right\}_{n \in \mathbb{N}}$ be a sequence of positive numbers less than 1 tending decreasingly to zero. For each $n \in \mathbb{N}$, there exists $\delta_{n}>0$ such that for each $h, k \in\left(0, \delta_{n}\right)$ if $f(h) \leq k \leq h$ or $f(k) \leq h \leq k$, then

$$
\frac{m_{2}\left(E_{n} \cap\{[-h, h] \times[-k, k]\}\right)}{4 h k}>1-\epsilon_{n} .
$$

Let $\left\{h_{n}\right\}_{n \in \mathbb{N}}$ be a sequence of positive numbers tending decreasingly to zero such that $h_{n} \in\left(0, \delta_{n}\right)$ for all $n \in \mathbb{N}$. Put

and

$$
l_{n}=\epsilon_{n} \cdot h_{n+1} \quad \text { for all } n \in \mathbb{N}
$$

$$
A=\bigcup_{n=1}^{\infty}\left(E_{n} \backslash\left\{\left[-l_{n}, l_{n}\right] \times\left[-l_{n}, l_{n}\right]\right\}\right) .
$$

We shall prove that $(0,0) \in D_{f}(A)$. Let $\epsilon>0$. Since $\epsilon_{n} \underset{n \rightarrow \infty}{\searrow} 0$, there exists $n_{0} \in \mathbb{N}$ such that $2 \epsilon_{n_{0}}<\epsilon$. Put $\delta=h_{n_{0}}$. Let $h, k \in(0, \delta)$ and assume that $f(h) \leq k \leq h$. There exists $n_{1} \in \mathbb{N}, n_{1} \geq n_{0}$ such that $h \in\left[h_{n_{1}+1}, h_{n_{1}}\right)$. 


\section{ELŻBIETA WAGNER-BOJAKOWSKA — WŁADYSŁAW WILCZYŃSKI}

Then,

$$
\begin{aligned}
& m_{2}(A \cap\{[-h, h] \times[-k, k]\}) \\
& \geq m_{2}\left(E_{n_{1}} \cap\{[-h, h] \times[-k, k]\}\right)-m_{2}\left(\left[-l_{n_{1}}, l_{n_{1}}\right] \times[-k, k]\right) \\
& =m_{2}\left(E_{n_{1}} \cap\{[-h, h] \times[-k, k]\}\right)-4 k \epsilon_{n_{1}} h_{n_{1}+1} .
\end{aligned}
$$

Simultaneously, $4 h k \geq 4 h_{n_{1}+1} k$ and $h, k \in\left(0, h_{n_{1}}\right) \subset\left(0, \delta_{n_{1}}\right)$, so

$$
\begin{aligned}
& \frac{m_{2}(A \cap\{[-h, h] \times[-k, k]\})}{4 h k} \\
& \geq \frac{m_{2}\left(E_{n_{1}} \cap\{[-h, h] \times[-k, k]\}\right)}{4 h k}-\frac{4 k \epsilon_{n_{1}} h_{n_{1}+1}}{4 h k} \\
& \geq 1-2 \epsilon_{n_{1}} \geq 1-2 \epsilon_{n_{0}}>1-\epsilon .
\end{aligned}
$$

If $f(k) \leq h \leq k$, then the proof is analogous. Consequently, $(0,0) \in D_{f}(A)$.

Theorem 5. A function $g: I \times I \rightarrow \mathbb{R}$ is $f$-approximately continuous if and only if for each $\alpha \in \mathbb{R}$ the sets

$$
E^{\alpha}=\left\{p \in \mathbb{R}^{2}: g(p)<\alpha\right\} \quad \text { and } \quad E_{\alpha}=\left\{p \in \mathbb{R}^{2}: g(p)>\alpha\right\}
$$

are of the type $F_{\sigma}$ and open in the topology $\mathcal{T}_{f}$.

Proof. Suppose that $g$ is $f$-approximately continuous. Fix $\alpha \in \mathbb{R}$. As $g$ is approximately continuous, the sets $E^{\alpha}$ and $E_{\alpha}$ are of the type $F_{\sigma}$. Observe that $E^{\alpha} \in \mathcal{T}_{f}$. Indeed, let $p_{0}=\left(x_{0}, y_{0}\right) \in E^{\alpha}$. Then, $g\left(p_{0}\right)<\alpha$ and there exists a measurable set $E$ such that $p_{0} \in E \cap D_{f}(E)$ and $g_{\mid E}$ is continuous at $p_{0}$. So, there exists $\delta>0$ such that

$$
g(p)<\alpha \quad \text { for } \quad p \in\left\{\left(x_{0}-\delta, x_{0}+\delta\right) \times\left(y_{0}-\delta, y_{0}+\delta\right)\right\} \cap E .
$$

Consequently,

and

$$
\left\{\left(x_{0}-\delta, x_{0}+\delta\right) \times\left(y_{0}-\delta, y_{0}+\delta\right)\right\} \cap E \subset E^{\alpha}
$$

$$
p_{0} \in D_{f}\left(\left\{\left(x_{0}-\delta, x_{0}+\delta\right) \times\left(y_{0}-\delta, y_{0}+\delta\right)\right\} \cap E\right),
$$

so $p_{0} \in D_{f}\left(E^{\alpha}\right)$. For the set $E_{\alpha}$, the proof is analogous.

Suppose now that for each $\alpha \in \mathbb{R}$ the sets $E^{\alpha}$ and $E_{\alpha}$ are of the type $F_{\sigma}$ and each point $p \in E^{\alpha}$ (resp. $E_{\alpha}$ ) is a density point of $E^{\alpha}$ (resp. $E_{\alpha}$ ) with respect to $f$. We shall prove that $g$ is $f$-approximately continuous on $I \times I$. Let $p_{0} \in I \times I$ and let $\epsilon$ be an arbitrary positive real number. To simplify the denotation, we may assume that $I=(-1,1)$ and $p_{0}=(0,0)$. Put

$$
E_{n}=\left\{p \in I \times I:|g(p)-g(0,0)|<\frac{1}{n}\right\} \quad \text { for each } n \in \mathbb{N} .
$$


Obviously,

$$
E_{n}=\left\{p \in I \times I: g(p)<g(0,0)+\frac{1}{n}\right\} \cap\left\{p \in I \times I: g(p)>g(0,0)-\frac{1}{n}\right\},
$$

so $E_{n} \subset D_{f}\left(E_{n}\right)$ and $(0,0) \in E_{n}$ for each $n \in \mathbb{N}$. Clearly, $\left\{E_{n}\right\}_{n \in \mathbb{N}}$ is a descending sequence of measurable sets such that $(0,0) \in D_{f}\left(E_{n}\right)$ for each $n \in \mathbb{N}$. By Lemma 1, there exists a strictly decreasing sequence $\left\{l_{n}\right\}_{n \in \mathbb{N}}$ of positive numbers tending to zero such that $(0,0)$ is a density point of a set

$$
A=\bigcup_{n=1}^{\infty}\left(E_{n} \backslash\left\{\left[-l_{n}, l_{n}\right] \times\left[-l_{n}, l_{n}\right]\right\}\right) \cup\{(0,0)\}
$$

with respect to $f$, so $(0,0) \in A \cap D_{f}(A)$ and $g_{\mid A}$ is continuous at $(0,0)$.

From the last theorem, it follows that each $f$-approximately continuous function is continuous with respect to the topology $\mathcal{T}_{f}$ in the domain.

Using Corollary 1, we can prove the next lemmas analogously to the proofs in [4, Lemma 11 and Lemma 12].

Lemma 2. Let $E$ be a set of the type $F_{\sigma}$ such that $E \subset D_{f}(E)$. There exists an $f$-approximately continuous function $g$ such that

(i) $0<g(p) \leq 1$ for $p \in E$,

(ii) $g(p)=0$ for $p \notin E$.

Lemma 3. Let $E_{1}, E_{2}, H$ be pairwise disjoint subsets of the plane such that $E_{1} \cup E_{2} \cup H=\mathbb{R}^{2}$ and $E_{1} \cup H, E_{2} \cup H$ are $\mathcal{T}_{f}$-open sets of the type $F_{\sigma}$. There exists $f$-approximately continuous function $g: \mathbb{R}^{2} \rightarrow \mathbb{R}$ such that

(i) $g(p)=0$ for $p \in E_{1}$,

(ii) $0<g(p)<1$ for $p \in H$,

(iii) $g(p)=1$ for $p \in E_{2}$.

From the last lemma we obtain the next result similarly as in [2] for ordinary density topology.

TheORem 6. The space $\left(\mathbb{R}^{2}, \mathcal{T}_{f}\right)$ is completely regular $\left(T_{3 \frac{1}{3}}\right)$.

Clearly, the space $\left(\mathbb{R}^{2}, \mathcal{T}_{f}\right)$ is not normal, analogously as the ordinary density topology on the plane. If $A$ and $B$ are two disjoint dense (in the Euclidean topology) subsets of the plane of measure zero, then no $f$-approximately function $g$ separates these sets, since each function separating $A$ and $B$ is discontinuous at each point and $g$ is Baire 1 .

Observe that each measurable subset of the plane is of the type $G_{\delta}$ in the topology $\mathcal{T}_{f}$. Let $A \in \mathcal{S}$. Then,

$$
A=\left(A \cap D_{f}(A)\right) \cup\left(A \backslash D_{f}(A)\right) .
$$




\section{ELŻBIETA WAGNER-BOJAKOWSKA — WŁADYSŁAW WILCZYŃSKI}

The first summand is the interior of $A$ in the topology $\mathcal{T}_{f}$ (see [3, Theorem 7]). From [3. Theorem 5], we have $m_{2}\left(A \backslash D_{f}(A)\right)=0$. Let $E$ be a set of the type $G_{\delta}$ (in the Euclidean topology) of measure zero such that $A \backslash D_{f}(A) \subset E$. Obviously,

$$
m_{2}\left(E \backslash\left(A \backslash D_{f}(A)\right)\right)=0, \quad A \backslash D_{f}(A)=E \backslash\left(E \backslash\left(A \backslash D_{f}(A)\right)\right)
$$

and $E \backslash\left(A \backslash D_{f}(A)\right)$ is closed in the topology $\mathcal{T}_{f}$. Consequently, $A \backslash D_{f}(A)$ is of the type $G_{\delta}$ in the topology $\mathcal{T}_{f}$, so $A$ is of this type, too.

Let $f, g \in C_{0}$ and $f(x) \leq g(x)$ for every $x \in(0,1]$. Then, for arbitrary measurable subset $A$ of the plane, $D_{f}(A) \subset D_{g}(A)$ and, consequently, $\mathcal{T}_{f} \subset \mathcal{T}_{g}$. In 3. we were looking for conditions under which two topologies $\mathcal{T}_{f}$ and $\mathcal{T}_{g}$ are equal. We proved that if $f \in C_{0}$ and $c \in(0,1]$, then $D_{f}(A)=D_{c f}(A)$ for $A \in \mathcal{S}$, so $\mathcal{T}_{f}=\mathcal{T}_{c f}$ (see [3, Theorem 3]. More generally, if

and

$$
f, g \in C_{0}, \quad f(x) \leq g(x) \quad \text { for every } \quad x \in(0,1]
$$

$$
\liminf _{x \rightarrow 0^{+}} \frac{f(x)}{g(x)}>0
$$

then $\mathcal{T}_{f}=\mathcal{T}_{g}(\underline{3}$, Theorem 17]). Particularly, if

then $\mathcal{T}_{f}=\mathcal{T}_{0}$.

$$
\liminf _{x \rightarrow 0^{+}} \frac{f(x)}{x}>0,
$$

Another condition sufficient for the equality $\mathcal{T}_{f}=\mathcal{T}_{g}$, which is described in terms of porosity, is given in [3, Theorem 20]. This theorem says that if $f, g \in C_{0}, g(x) \leq f(x)$ for every $x \in(0,1]$ and for each decreasing sequence $\left\{c_{n}\right\}_{n \in \mathbb{N}}$ convergent to zero

$$
\lim _{n \rightarrow \infty} p^{+}\left(M_{c_{n}}, 0\right)=0
$$

where $M_{c}=\{x \in(0,1]: g(x) \geq c \cdot f(x)\}$ for $c \in(0,1)$ and $p^{+}(A, x)$ denotes the right-hand porosity of $A$ at $x$, then $\mathcal{T}_{f}=\mathcal{T}_{g}$.

Here, we give some condition, which is necessary for the equality $\mathcal{T}_{f}=\mathcal{T}_{g}$ for arbitrary functions $f, g \in C_{0}$ such that $g(x) \leq f(x)$ for every $x \in(0,1]$. We also prove that this condition is sufficient if $g$ is a strictly increasing function.

Let $f, g \in C_{0}$ be such that $g(x) \leq f(x)$ for every $x \in(0,1]$. Let $x \in(0,1]$ and $n \in \mathbb{N}$. If $g(x) \leq f(x) / n$, then put

$$
z_{n}(x)=\min \left\{\sup \left\{z \in[x, 1]: g(z) \leq \frac{1}{n} f(x)\right\}, 2 x\right\} .
$$

If $g(x)>f(x) / n$, then $z_{n}(x)=x$.

Let $n$ be a fixed positive integer, and let $f, g \in C_{0}$. If there exists $\delta>0$ such that

$$
\frac{1}{n} f(x) \leq g(x) \leq f(x) \quad \text { for every } \quad x \in(0, \delta],
$$


then

$$
D_{\frac{1}{n} f}(A) \subset D_{g}(A) \subset D_{f}(A) \quad \text { for every } \quad A \in \mathcal{S} .
$$

Simultaneously, $D_{\frac{1}{n} f}(A)=D_{f}(A)$ by [3, Theorem 3] for every $A \in \mathcal{S}$, so $D_{f}(A)=D_{g}(A)$ for every $A \in \mathcal{S}$ and $\mathcal{T}_{f}=\mathcal{T}_{g}$.

Definition 3. Let $f, g \in C_{0}$ be such that $g(x) \leq f(x)$ for every $x \in(0,1]$. We shall say that the pair $(f, g)$ fulfills condition (W) if and only if, for each real number $a>0$, there exist $n \in \mathbb{N}$ and $\delta>0$ such that

$$
\frac{z_{n}(x)-x}{z_{n}(x)} \leq a \quad \text { for every } \quad x \in(0, \delta) .
$$

TheOREM 7. Let $f, g \in C_{0}$ be such that $g(x) \leq f(x)$ for every $x \in(0,1]$ and for each $\delta>0$ and each $n \in \mathbb{N}$ there exists $x \in(0, \delta)$ such that $g(x)<f(x) / n$. If $D_{g}(A)=D_{f}(A)$ for arbitrary $A \in \mathcal{S}$, then the pair $(f, g)$ fulfills condition $(\mathrm{W})$.

P r o of. Suppose, on the contrary, that there exists a number $a>0$ such that for each $n \in \mathbb{N}$ and $\delta>0$ there exists a point $x \in(0, \delta)$ for which

$$
\frac{z_{n}(x)-x}{z_{n}(x)}>a .
$$

We shall prove that $D_{f}(A) \neq D_{g}(A)$ for some $A \in \mathcal{S}$. For this purpose, we construct a set $A \subset[0,1] \times[0,1]$ such that $(0,0)$ is a dispersion point of $A$ with respect to $f$ and is not a dispersion point of $A$ with respect to $g$.

First, by easy induction, we define a sequence $\left\{x_{n}\right\}_{n \in \mathbb{N}}$ such that

Obviously,

$$
\frac{z_{n}\left(x_{n}\right)-x_{n}}{z_{n}\left(x_{n}\right)}>a \text { and } \quad x_{n+1}<\frac{1}{2} x_{n} \quad \text { for every } n \in \mathbb{N} .
$$

$$
z_{n}\left(x_{n}\right) \neq x_{n}
$$

so

$$
g\left(z_{n}\left(x_{n}\right)\right) \leq \frac{1}{n} f\left(x_{n}\right) \quad \text { and } \quad z_{n}\left(x_{n}\right) \leq 2 x_{n} \quad \text { for every } \quad n \in \mathbb{N} .
$$

Put

$$
A=\bigcup_{n=1}^{\infty}\left\{\left[x_{n}, z_{n}\left(x_{n}\right)\right] \times\left[0, g\left(z_{n}\left(x_{n}\right)\right)\right]\right\} .
$$

Obviously,

$$
\begin{aligned}
& \frac{m_{2}\left(A \cap\left\{\left[0, z_{n}\left(x_{n}\right)\right] \times\left[0, g\left(z_{n}\left(x_{n}\right)\right)\right]\right\}\right)}{m_{2}\left(\left[0, z_{n}\left(x_{n}\right)\right] \times\left[0, g\left(z_{n}\left(x_{n}\right)\right)\right]\right)} \\
& >\frac{\left(z_{n}\left(x_{n}\right)-x_{n}\right) \cdot g\left(z_{n}\left(x_{n}\right)\right)}{z_{n}\left(x_{n}\right) \cdot g\left(z_{n}\left(x_{n}\right)\right)}=\frac{z_{n}\left(x_{n}\right)-x_{n}}{z_{n}\left(x_{n}\right)}>a
\end{aligned}
$$

for every $n \in \mathbb{N}$, so $(0,0)$ is not a dispersion point of $A$ with respect to $g$. 


\section{ELŻBIETA WAGNER-BOJAKOWSKA — WŁADYSŁAW WILCZYŃSKI}

Fix $n \in \mathbb{N}$. For $t \in\left[z_{n}\left(x_{n}\right), x_{n-1}\right]$ and $y \in[f(t), t]$, using (2), we have

because

$$
\frac{m_{2}(A \cap\{[0, t] \times[0, y]\})}{m_{2}([0, t] \times[0, y])} \leq \frac{z_{n}\left(x_{n}\right) \cdot g\left(z_{n}\left(x_{n}\right)\right)}{z_{n}\left(x_{n}\right) \cdot f\left(z_{n}\left(x_{n}\right)\right)} \leq \frac{\frac{1}{n} f\left(x_{n}\right)}{f\left(x_{n}\right)}=\frac{1}{n}
$$

$$
g\left(z_{n}\left(x_{n}\right)\right) \leq \frac{1}{n} f\left(x_{n}\right) \quad \text { and } \quad x_{n} \leq z_{n}\left(x_{n}\right) .
$$

For $t \in\left[x_{n}, z_{n}\left(x_{n}\right)\right]$ and $y \in[f(t), t]$, using (2), we obtain

$$
\frac{m_{2}(A \cap\{[0, t] \times[0, y]\})}{m_{2}([0, t] \times[0, y])} \leq \frac{z_{n}\left(x_{n}\right) \cdot g\left(z_{n}\left(x_{n}\right)\right)}{x_{n} \cdot f\left(x_{n}\right)} \leq \frac{2 \cdot \frac{1}{n} f\left(x_{n}\right)}{f\left(x_{n}\right)}=\frac{2}{n}
$$

because

$$
z_{n}\left(x_{n}\right) \leq 2 x_{n} \quad \text { and } \quad g\left(z_{n}\left(x_{n}\right)\right) \leq \frac{1}{n} f\left(x_{n}\right) .
$$

If $[0, t] \times[0, y]$ is such that $f(y) \leq t \leq y$, then

$$
A \cap\{[0, t] \times[0, y]\}=A \cap\{[0, t] \times[0, t]\},
$$

so

and finally,

$$
\frac{m_{2}(A \cap\{[0, t] \times[0, y]\})}{m_{2}([0, t] \times[0, y])} \leq \frac{m_{2}(A \cap\{[0, t] \times[0, t]\})}{m_{2}([0, t] \times[0, t])},
$$

$$
\frac{m_{2}(A \cap\{[0, t] \times[0, y]\})}{m_{2}([0, t] \times[0, y])} \leq \frac{1}{n} \quad \text { for } \quad t \in\left[z_{n}\left(x_{n}\right), x_{n-1}\right]
$$

and

$$
\frac{m_{2}(A \cap\{[0, t] \times[0, y]\})}{m_{2}([0, t] \times[0, y])} \leq \frac{2}{n} \quad \text { for } \quad t \in\left[x_{n}, z_{n}\left(x_{n}\right)\right] .
$$

Since $n \in \mathbb{N}$ was arbitrary, $(0,0)$ is a dispersion point of $A$ with respect to $f$.

Theorem 8. Let $f, g \in C_{0}$ be such that $g(x) \leq f(x)$ for every $x \in(0,1]$ and for each $\delta>0$ and each $n \in \mathbb{N}$ there exists $x \in(0, \delta)$ such that $g(x)<f(x) / n$. If $g$ is a strictly increasing function and the pair $(f, g)$ fulfills condition (W), then $D_{g}(A)=D_{f}(A)$ for every $A \in \mathcal{S}$.

P r o of. Obviously, $D_{g}(A) \subset D_{f}(A)$ for every $A \in \mathcal{S}$. Now, we shall prove that if $g$ is strictly increasing and condition (W) is fulfilled, then $D_{f}(A) \subset D_{g}(A)$ for every $A \in \mathcal{S}$.

Let $A \in \mathcal{S}$ and let $(0,0)$ be a dispersion point of $A$ with respect to $f$. We shall prove that $(0,0)$ is a dispersion point of $A$ with respect to $g$, i.e., for each $\epsilon>0$ there exists $\eta>0$ such that for each $x, y \in(0, \eta)$ if $g(x) \leq y<f(x)$ or $g(y) \leq x<f(y)$, then

$$
\frac{m_{2}(A \cap\{[-x, x] \times[-y, y]\})}{4 x y}<\epsilon
$$

(if $f(x) \leq y \leq x$ or $f(y) \leq x \leq y$, then the inequality follows from our assumption). 
Let $\epsilon>0$. We may assume that $\epsilon<1$. Put $a=\epsilon / 2$. From (W), there exist a positive integer $n_{0}$ and a positive real number $\delta_{1}<1 / 2$ such that for each $x \in\left(0, \delta_{1}\right)$ we have

$$
\frac{z_{n_{0}}(x)-x}{z_{n_{0}}(x)}<\frac{\epsilon}{2} .
$$

Then, $z_{n_{0}}(x)-x<z_{n_{0}}(x) / 2$, so $x \leq z_{n_{0}}(x)<2 x<1$, and consequently, from definition of $z_{n_{0}}(x)$ and the continuity of $f$ and $g$, we have $g\left(z_{n_{0}}(x)\right)=f(x) / n_{0}$ for every $x \in\left(0, \delta_{1}\right)$.

Since $(0,0)$ is a dispersion point of $A$ with respect to $f$, there exists $\delta_{2}>0$ such that for each $x \in\left(0, \delta_{2}\right)$ and $y \in[f(x), x]$

$$
\frac{m_{2}(A \cap\{[-x, x] \times[-y, y]\})}{4 x y}<\frac{\epsilon}{2 n_{0}} .
$$

Put $\delta=\min \left(\delta_{1}, \delta_{2} / 2\right)$. Let $x \in(0, \delta)$. Then, $z_{n_{0}}(x)<2 x<\delta_{2}$.

First, we shall prove that the required condition holds for the point $\left(z_{n_{0}}(x), y\right)$, where $y \in\left[g\left(z_{n_{0}}(x)\right), f\left(z_{n_{0}}(x)\right)\right)$. There are three cases:

$\mathbf{1}^{\circ} g\left(z_{n_{0}}(x)\right) \leq y<f(x)$. Then using (4) for $y=f(x)$ and (3), we obtain

$$
\begin{aligned}
& \frac{m_{2}\left(A \cap\left\{\left[-z_{n_{0}}(x), z_{n_{0}}(x)\right] \times[-y, y]\right\}\right)}{4 z_{n_{0}}(x) y} \\
& \leq \frac{m_{2}(A \cap\{[-x, x] \times[-y, y]\})}{4 z_{n_{0}}(x) g\left(z_{n_{0}}(x)\right)}+\frac{4\left(z_{n_{0}}(x)-x\right) y}{4 z_{n_{0}}(x) y} \\
& \leq n_{0} \cdot \frac{m_{2}(A \cap\{[-x, x] \times[-y, y]\})}{4 x f(x)}+\frac{z_{n_{0}}(x)-x}{z_{n_{0}}(x)}<\epsilon
\end{aligned}
$$

since $g\left(z_{n_{0}}(x)\right)=f(x) / n_{0}$ and $x \leq z_{n_{0}}(x)$.

$\mathbf{2}^{o} f(x) \leq y \leq x$. Then from (4) and (3), we obtain

$$
\begin{aligned}
& \frac{m_{2}\left(A \cap\left\{\left[-z_{n_{0}}(x), z_{n_{0}}(x)\right] \times[-y, y]\right\}\right)}{4 z_{n_{0}}(x) y} \\
& \leq \frac{m_{2}(A \cap\{[-x, x] \times[-y, y]\})}{4 z_{n_{0}}(x) y}+\frac{4\left(z_{n_{0}}(x)-x\right) y}{4 z_{n_{0}}(x) y} \\
& \leq \frac{m_{2}(A \cap\{[-x, x] \times[-y, y]\})}{4 x y}+\frac{z_{n_{0}}(x)-x}{z_{n_{0}}(x)}<\epsilon
\end{aligned}
$$

since $x \leq z_{n_{0}}(x)$. 


\section{ELŻBIETA WAGNER-BOJAKOWSKA — WŁADYSŁAW WILCZYŃSKI}

$\mathbf{3}^{o} x \leq y<f\left(z_{n_{0}}(x)\right)$. Then using (4) for $y=x$ and (3), we obtain

$$
\begin{aligned}
& \frac{m_{2}\left(A \cap\left\{\left[-z_{n_{0}}(x), z_{n_{0}}(x)\right] \times[-y, y]\right\}\right)}{4 z_{n_{0}}(x) y} \\
& \leq \frac{m_{2}(A \cap\{[-y, y] \times[-y, y]\})}{4 z_{n_{0}}(x) y}+\frac{4\left(z_{n_{0}}(x)-y\right) y}{4 z_{n_{0}}(x) y} \\
& \leq \frac{m_{2}(A \cap\{[-y, y] \times[-y, y]\})}{4 y^{2}}+\frac{z_{n_{0}}(x)-x}{z_{n_{0}}(x)}<\epsilon
\end{aligned}
$$

since $x \leq y<f\left(z_{n_{0}}(x)\right) \leq z_{n_{0}}(x)$.

Consequently, if $x \in(0, \delta)$, then

$$
\frac{m_{2}\left(A \cap\left[-z_{n_{0}}(x), z_{n_{0}}(x)\right] \times[-y, y]\right)}{4 z_{n_{0}}(x) y}<\epsilon
$$

for each $y$ fulfilling inequalities $g\left(z_{n_{0}}(x)\right) \leq y \leq z_{n_{0}}(x)$ (for $y \in\left[f\left(z_{n_{0}}(x)\right), z_{n_{0}}(x)\right]$ it follows immediately from (4)).

Let us consider the interval $(0, \delta)$. From our assumption, there exists a point $\eta \in(0, \delta)$ such that $g(\eta)<f(\eta) / n_{0}$.

We shall prove that for each point $t \in(0, \eta)$ there exists $x \in(0, t]$ such that $z_{n_{0}}(x)=t$. Let $t \in(0, \eta)$. Then, $0<g(t)<g(\eta)<f(\eta) / n_{0}$. The function $f / n_{0}$ is continuous, so there exists $x \in(0, \eta)$ such that

$$
\frac{1}{n_{0}} f(x)=g(t)
$$

Simultaneously, since $x<\eta<\delta \leq \delta_{1}$, we have $x \leq z_{n_{0}}(x)<2 x<1$ and

$$
z_{n_{0}}(x)=\sup \left\{z \geq x: g(z) \leq \frac{1}{n_{0}} f(x)\right\} .
$$

Obviously, $g(t) \leq f(x) / n_{0}$ and $t \geq x$, so $t \in\left\{z \geq x: g(z) \leq f(x) / n_{0}\right\}$. Since $g$ is strictly increasing, we have $g\left(t_{1}\right)>g(t)=f(x) / n_{0}$ for $t_{1}>t$. Consequently,

$$
z_{n_{0}}(x)=t .
$$

Finally, let

$$
t \in(0, \eta) \text { and } g(t) \leq y \leq t .
$$

There exists $x \in(0, \eta)$ such that $z_{n_{0}}(x)=t$. From (5), we obtain

$$
\frac{m_{2}(A \cap\{[-t, t] \times[-y, y]\})}{4 t y}=\frac{m_{2}\left(A \cap\left\{\left[-z_{n_{0}}(x), z_{n_{0}}(x)\right] \times[-y, y]\right\}\right)}{4 z_{n_{0}}(x) y}<\epsilon .
$$

We can obtain analogous inequality if $y \in(0, \eta)$ and $g(y) \leq x \leq y$. Consequently, $(0,0)$ is a dispersion point of $A$ with respect to $g$. 
DENSITY TOPOLOGIES ON THE PLANE BETWEEN ORDINARY AND STRONG. II

\title{
REFERENCES
}

[1] BRUCKNER, A. M.: Differentiation of Real Functions, in: Lecture Notes in Math., Vol. 659, Springer-Verlag, Berlin, 1978.

[2] GOFFman, C.-NEUGEBAUER, C. J.-NISHIURA, T.: Density topology and approximate continuity, Duke Math. J. 28 (1961), 497-505.

[3] WAGNER-BOJAKOWSKA, E.-WILCZYŃSKI, W.: Density topologies on the plane between ordinary and strong, Tatra Mt. Math. Publ. 44 (2009), 139-151.

[4] ZAHORSKI, Z.: Sur la prémière dérivée, Trans. Amer. Math. Soc. 69 (1950), 1-54.

Received November 28, 2013

\author{
University of Łódź \\ Faculty of Mathematics \\ and Computer Science \\ Banacha 22 \\ PL-90-238 Łódź \\ POLAND
}

E-mail: wagner@math.uni.lodz.pl wwil@uni.lodz.pl 